\title{
Language constructs as compatibility intervals: a small-scale experiment
}

\author{
Pavlo Kapustin ${ }^{1}$, Michael Kapustin ${ }^{2}$ \\ ${ }^{1}$ Department of Information Science and Media Studies, University of Bergen, Norway \\ 2Department of General Physics, Moscow Institute of Physics and Technology, Russia \\ https://doi.org/10.36505/ExLing-2020/11/0025/000440
}

\begin{abstract}
We conducted a small-scale experiment in which the respondents were asked to relate different language constructs (e.g. "possible", "afterwards", "seldom") to numbers on a certain scale (e.g. surprisingness, time, frequency). We studied how the respondents expressed vagueness of the meaning using a numeric scale, examined how their answers related to scalar implicatures and questioned whether the meaning of vague constructs could be adequately modelled using compatibility intervals, a meaning representation (computational model) we recently proposed.
\end{abstract}

Keywords: scales, vague constructs, meaning representations.

\section{Introduction}

Using numeric approaches for modelling meaning of vague constructs has been described, for example with use of relatively well-known fuzzy sets (Zadeh, 1971) or compatibility intervals (Kapustin and Kapustin, 2019a) that are closely related to fuzzy sets. Both meaning representations are based on the notion of compatibility of a given language construct (e.g. "young") with the values of a certain property (e.g. age) on a certain scale (e.g. 0-100).

While there are some examples in the literature that use these meaning representations, there is very little experimental work on the subject. Some studies show how such representations may be derived from experiments, e.g. (Hersh and Caramazza, 1976), however, to the best of our knowledge, no experiments aiming to directly express vagueness of meaning in a response using numbers have been performed.

We conducted a small-scale experiment exploring one possible approach to gathering such data. The experiment had the following goals:

- Study how the respondents would express vagueness of meaning using a numeric scale.

- Study how the respondents would compare meaning of related constructs using a numeric scale.

- Study whether the answers agree with scalar implicatures where appropriate.

- Study whether the meaning of vague constructs could be adequately modelled using compatibility intervals.

ExLing 2020: Proceedings of $11^{\text {th }}$ International Conference of Experimental Linguistics, 12-14 October 2020, Athens, Greece 


\section{Compatibility intervals}

Compatibility intervals consist of three number intervals: left ("increasing"), main and right ("decreasing"). For example, one could define the compatibility interval for "young" over property "age" as [0-18 - 30-50] (which is, of course, entirely subjective). This says that description "young" is compatible with ages 18-30, incompatible with ages over 50, and that the compatibility increases between ages 0 and 18 and decreases between ages 30 and 50. We use double hyphens between the start and the end of the main subinterval, and single hyphens between the start and the end of the left and the right subintervals.

Study design

We used an online survey to interview 22 respondents about 20 language constructs, asking the respondents to relate the constructs to 5 different properties (see Table 1). Possible answers were represented as numbered check boxes arranged horizontally to form a numeric scale. For each construct, the respondents were asked two questions, first focusing on the normal meaning of the construct and second focusing on a somewhat broader meaning of the construct (see an example question below). When it comes to the dimensions of meaning, we used one-dimensional projections, following some of the examples from Kapustin and Kapustin (2019b).

\section{Properties and scales}

Table 2. Properties and scales.

\begin{tabular}{|l|l|}
\hline Property & Scale (possible answers) \\
\hline Time & 0 (right now) - 9 (in the distant future) \\
\hline Surprisingness & $\begin{array}{l}0 \text { (completely anticipated) - } \\
\text { surprising) }\end{array}$ \\
\hline Perceived duration & $\begin{array}{l}0 \text { (instantaneous), 1(much shorter than expected), } \\
2,3,4,5 \text { (as expected), 6, 7, 8, 9 (much longer } \\
\text { than expected) }\end{array}$ \\
\hline Event frequency & 0 (never) - 9 (extremely often) \\
\hline $\begin{array}{l}\text { Event expectedness (probability in } \\
\text { given conditions) }\end{array}$ & 0 (not expected at all) - 9 (certain to happen) \\
\hline
\end{tabular}

\section{Example question}

Someone describes how often a certain event happens by using the following words. According to this description, how often can the event happen? Please select the choices that fit (0 means "never", 9 means "extremely often", and 1-8 correspond to everything in between).

a) According to the description "seldom", this event can occur: 0 (never), 1, 2, $3,4,5,6,7,8,9$ (extremely often).

b) Somewhat less likely, the description "seldom" can also include events that occur: 0 (never), 1, 2, 3, 4, 5, 6, 7, 8, 9 (extremely often). 


\section{Results}

We found that the majority of the responses formed coherent intervals, and most of them were also representable as compatibility intervals, e.g. see examples 1-3 in Table 2. While interesting, we think this is largely explained by the design of the experiment.

Some responses, however, could not be represented as compatibility intervals. In example 4 in Table 2, the answers to the first and second questions are far apart, possibly suggesting that the respondent has two different meanings of "entire" in mind. In example 5 in Table 2, the answer to the second question is a "shift" of the answer to the first question, possibly suggesting that the respondent gives an alternative, less "polar" meaning of "occasionally" as the answer to the second question. In total, there were very few answers similar to the last two examples. We believe this is partly because the phrasing of the questions implied that the answer to the second question should be a "wider" version of the same meaning of the construct.

Most of the respondents found that "seldom" is not compatible with events that never happen, always is not compatible with events that always happen, and "mere", "just" and "only" are not compatible with things that happen instantaneously (a somewhat invented example of such use would be "it only took me zero minutes to get there"). This agrees with scalar implicatures.

According to averaged responses, "later" [2.69-3.25-7.00-7.69] and "eventually" [3.76-5.12-8.35-8.53] have a wider spread than "afterwards" [1.131.60-4.13-5.00], and "eventually" spans further in the future than "later". Also, "regularly" seems to be a more concrete word in terms of event expectedness (probability in given conditions) than in terms of event frequency (averaged intervals [3.67-4.60-7.00-7.67] for frequency and [4.21-4.79-6.57-7.21] for expectedness). Also, "entire" [4.93-5.47-7.00-7.40] is a stronger construct than "whole" [4.80-5.07-6.33-6.80], when related to perceived duration.

Table 3. Answer examples.

\begin{tabular}{|l|l|l|}
\hline & Construct & Property \\
\hline 1 & $\begin{array}{l}\text { In a while } \\
\text { First question: 3, 4, 5, 6. Second question: 2, 7, 8 } \\
\text { Compatibility interval: [2-3-6-8] }\end{array}$ & Time \\
\hline 2 & $\begin{array}{l}\text { Possible } \\
\text { First question: 4, 5. Second question: 3, 4, 5, 6 } \\
\text { Compatibility interval: [3-4-5-6] }\end{array}$ & Surprisingness \\
\hline 3 & $\begin{array}{l}\text { Entire (as in “an entire minute") } \\
\text { First question: 7, 8, 9. Second question: 5, 6 } \\
\text { Compatibility interval: [5-7-9] }\end{array}$ & Perceived duration \\
\hline 4 & $\begin{array}{l}\text { Entire (as in "an entire minute") } \\
\text { First question: 5. Second question: 9 }\end{array}$ & Perceived duration \\
\hline 5 & $\begin{array}{l}\text { Occasionally } \\
\text { First question: 3, 4, 5. Second question: 4, 5,6 }\end{array}$ & Event frequency \\
\hline
\end{tabular}




\section{Discussion}

Despite the limitations of the current survey, we think that the results of the experiment are interesting. We find compatibility intervals to be a promising and relatively easily linguistically interpretable meaning representation that could be used for computational purposes. While specific compatibility intervals have no significance and are to a large degree context dependent, it is interesting how they compare to each other in individual responses, allowing to express certain aspects of the meaning of the language constructs in a quantitative, measurable way. We also think this perspective shows that such properties as vagueness and mirativity (Zeevat, 2013) may apply to more constructs than often considered, especially taking into account that many constructs have different dimensions of meaning, conveying information about different properties.

We think that further and improved experiments of this kind are interesting both from the computational and the theoretical perspectives. In the future, we would like to work on improving the survey, for example, add self-assessment of question understanding, randomize question order, use more constructs, more respondents and require the respondents to be native speakers of the survey language.

\section{References}

Hersh, H.M., Caramazza, A., 1976. A fuzzy set approach to modifiers and vagueness in natural language. Journal of Experimental Psychology: General 105, 254.

Kapustin, P., Kapustin, M., 2019a. Modeling language constructs with compatibility intervals, in: Proceedings of the IWCS 2019 Workshop on Computing Semantics with Types, Frames and Related Structures. pp. 49-54.

Kapustin, P., Kapustin, M., 2019b. Modeling language constructs with fuzzy sets: some approaches, examples and interpretations, in: Proceedings of the 13th International Conference on Computational Semantics-Student Papers. pp. 24-33.

Zadeh, L.A., 1971. Quantitative fuzzy semantics. Information sciences 3, 159-176.

Zeevat, H., 2013. Expressing surprise by particles, in: Beyond Expressives: Explorations in Use-Conditional Meaning. Brill, pp. 297-320. 